\title{
Arrhythmias and \\ COVID-19 infection
}

\section{Ashley Chin*, Jane Moses" and Andrew Thornton ${ }^{\dagger}$}

"Cardiac Clinic, University of Cape Town and Groote Schuur

Hospital, Observatory, Cape Town, South Africa

"Stellenbosch University and Tygerberg Hospital, Bellville,

South Africa

tSunninghill Hospital, Johannesburg, South Africa

Address for correspondence:

Associate Professor Ashley Chin

Cardiac Clinic

University of Cape Town and Groote Schuur Hospital

Anzio Road

Observatory

7925

South Africa

Email:

Ashley.chin@uct.ac.za

\section{INTRODUCTION}

Coronavirus disease 2019 (COVID-19), caused by severe acute respiratory syndrome coronavirus 2 (SARS-CoV-2), is a global pandemic and has affected around 5.8 million people worldwide with over 360000 deaths between 31 December 2019 and I June 2020.(1) Cardiovascular manifestations of COVID-19 include acute coronary syndromes, heart failure, thromboembolic events, cardiogenic shock, cardiac arrest and arrhythmias.(2) This review will focus on the epidemiology, challenges to the management of arrhythmias in patients with COVID-19, and highlight the potential risks of novel treatments that prolong the QT interval.

\section{EPIDEMIOLOGY}

The incidence of arrhythmias in patients with COVID-19 is unknown. There is preliminary evidence that palpitations and arrhythmias are a common presenting symptom or manifestation of COVID-19 infection. In a study from Wuhan, China, 10 (7.3\%) of 137 patients had reported palpitations as an initial symptom. ${ }^{(3)}$ In another study of 138 patients hospitalised with COVID-19, arrhythmias (unspecified) were reported in 23 ( $16.7 \%)$ patients -7 (7\%) patients in the non-ICU setting versus 16 (44\%) in patients admitted to ICU. ${ }^{(4)}$

\section{ABSTRACT}

Arrhythmias can be a clinical manifestation of COVID-19 infection. COVID-19 infection can also be a precipitant of known arrhythmias which can increase the risk of morbidity and mortality. Management of arrhythmias should generally follow standard guidelines of arrhythmia management. Catheter ablation in the midst of the COVID-19 pandemic should be limited to arrhythmias that are immediately life-threatening or which may affect prognosis in the short-term. Some novel treatments like chloroquine, hydroxychloroquine and azithromycin can prolong the QT interval and predispose patients to life-threatening arrhythmias. SAHeart 2020; 17:362-369

\section{CHALLENGES TO ARRHYTHMIA MANAGEMENT IN PATIENTS WITH COVID- I 9}

COVID-19 brings some unique challenges to arrhythmia management. These challenges are somewhat different to traditional arrhythmia management, depending on the clinical situation. There are patients with arrhythmias caused by COVID-19 (either by the infection itself or therapies given), and those with underlying arrhythmias (in either structurally normal or abnormal hearts) who are concurrently infected with COVID-19. There is some overlap between these 2 groups.

As with many other facets of the disease, infection with COVID- 19 can cause a variety of arrhythmias. The most feared of these are malignant ventricular arrhythmias (ventricular tachycardia and fibrillation), which can be found in 5.9\% of cases. ${ }^{(6)}$ Postulated mechanisms include the high level of circulating cytokines (particularly IL-6) seen in severe cases of COVID- 19 infection, hypoxia-induced apoptosis and COVID- 19 myocarditis, all of which may result in myocardial damage and cause atrial or ventricular arrhythmias. (7) The management of these arrhythmias is influenced by the high risk environment in which they occur.

\section{ARRHYTHMIA MANAGEMENT IN COVID- 19 POSITIVE PATIENTS}

SARS-CoV-2 is a highly infectious virus which can be exacerbated by aerosolisation of viral particles with high flow oxygen, bronchoscopy, open tracheal suctioning, intubation, extubation, non-invasive positive pressure ventilation, en- 
doscopy, or transoesophageal echocardiography. It is recommended that as few healthcare practitioners as possible be exposed to clinically high risk areas, by taking measures such as limiting the number of people on ward rounds. ${ }^{(8)}$ This recommendation extends to referrals and consultations, where the recommendation is that as little time be spent face-to-face at the patient's bedside as possible, with most electrophysiology (EP) referrals being managed by reviewing the patient's chart and monitoring data including the ECG. ${ }^{(8)}$

As ICUs and other high-care facilities where COVID- 19 patients are being treated become overwhelmed, the first consideration is to decide which patients need continuous cardiac monitoring. It is recommended that a baseline history, examination and risk assessment for arrhythmic events be performed on all patients. The exact contribution of COVID-19 to arrhythmia development in patients who are asymptomatic or only mildly symptomatic is not known. In those patients who are ill enough to be admitted to hospital, it is recommended that a baseline ECG (ideally a 12 lead ECG, but a single or multiple lead ECG from telemetry will suffice if normal) should be obtained on admission to hospital (unless a baseline ECG taken within the last month is available, in which case this should be used).(9) Hypoxia and electrolyte abnormalities are known to contribute to arrhythmia risk and it is recommended that the $\mathrm{K}^{+}$be maintained at above $4 \mathrm{mmol} / \mathrm{l}$ and the $\mathrm{Mg}^{2+}$ be maintained at above $2 \mathrm{mg} / \mathrm{dl}^{\left({ }^{(9)}\right.}$ In the absence of documented cardiac arrhythmias, suspected myocardial ischemia, or other standard indications, continuous ECG monitoring is not required. If available, continuous ECG monitoring can be used in lieu of repeated 12 lead ECGs to minimise staff exposure to patients and to preserve personal protective equipment (PPE).(10)

For the diagnosis of arrhythmias, a 12 lead ECG is considered the gold standard but a rhythm strip can also be used. The most common arrhythmia in patients with COVID-19 is sinus tachycardia, but the most common pathologic arrhythmias include atrial fibrillation (AF), atrial flutter (AFL), monomorphic ventricular tachycardia (MMVT) and polymorphic ventricular tachycardia (PMVT). ${ }^{(10)}$

The management of specific arrhythmias in a patient with COVID-19 infection is similar to that of patients without COVID-19 infection. In a patient with a haemodynamically unstable arrhythmia (whether it be supraventricular or ventricular), external direct current (DC) cardioversion is the treatment of choice, followed by the initiation of medical therapy as appropriate. In patients who are haemodynamically stable, the recommendation is that medical therapy be initiated according to standard guidelines for that condition (please refer to the relevant guidelines). In stable patients who respond to medical therapy, catheter ablation (if indicated) should be postponed until such time as the patient has recovered from COVID-19 infection. ${ }^{(8)}$

\section{A few specific aspects of management deserve special attention:}

Torsades de Pointes (TdP):

- For patients with a single episode of TdP, treatment with IV magnesium along with correction of metabolic/electrolyte derangements and/or stopping all QT-prolonging drugs may be sufficient. The patient should be carefully monitored until electrolytes are normalised and the QT interval normalises. This is particularly relevant in patients taking chloroquine, hydroxychloroquine or a macrolide antibiotic as novel therapy for COVID-19 (see below).

- For patients with multiple self-terminating episodes of TdP, the same therapies are utilised as in patients with a single episode (i.e. IV magnesium, correction of metabolic/ electrolyte derangements, and/or removal of any inciting medications), along with additional interventions to increase the heart rate, which include overdrive pacing and/or IV isoproterenol infusion (where available). ${ }^{(10)}$

Atrial fibrillation (AF) and atrial flutter (AFL):

- Rate control with B-blockers or non-dihydropyridine calcium channel blockers should be first-line therapy.

- Anticoagulation should be considered according to the CHA2DS2-VASc score as per standard practice.

- Consideration should be given to stopping anti-arrhythmic drugs with QT-prolonging effects, should specific therapies for COVID-19, which also prolong the QT, be necessary.

- The combination of amiodarone with hydroxychloroquine and azithromycin should be avoided.

- Transoesophageal echocardiography (TOE) should be avoided as far as possible, with elective cardioversion either being delayed until the patient has recovered from COVID-19 infection, or after 3 weeks of effective anticoagulation.

A definitive rate or rhythm control strategy (including the potential for catheter ablation), should be reviewed once the COVID- 19 infection has resolved.(2)

\section{Monomorphic ventricular tachycardia (MMVT):}

- A point of care transthoracic echocardiogram should be considered.

- Coronary angiography or other investigations should be considered based on the clinical likelihood of an acute coronary syndrome, myocarditis or cardiomyopathy. 
Amiodarone is the drug of choice, should the patient have recurrent MMVT.

- Caution should be exercised in adding amiodarone to other therapies that prolong the QT (review the necessity for other QT-prolonging medications).

- The need for implantation of a secondary prevention implantable cardioverter-defibrillator (ICD) should be reviewed once the COVID-19 infection has cleared. ${ }^{(2)}$

Paroxysmal supra-ventricular tachycardias (SVT):

Vagal manoeuvres and adenosine can be safely applied.

Medical therapy with B-blockers (or non-dihydropyridine calcium channel blockers where B-blockers are contraindicated) should be initiated.

The indication for catheter ablation should be assessed after the COVID-19 pandemic.(2)

Channelopathies:

Patients with inherited arrhythmia syndromes (like congenital Long QT syndrome (LQTS) and Brugada syndrome) are potentially at increased risk of ventricular arrhythmias and sudden death with COVID-19 infection. (11)

- Congenital LQTS patients with COVID-19 are potentially at increased risk of TdP/VF because of other factors that prolong the QT interval. Factors include electrolyte abnormalities (hypokalaemia, hypomagnesaemia and hypocalcaemia), use of QT-prolonging drugs and liver and renal disease. LQTS type 2 patients may also be at increased risk because of fever (similar to Brugada syndrome - see below). The use of any novel therapies for COVID-19 which prolong the QT interval should not be used in patients with congenital LQTS. ${ }^{(1)}$

- Brugada syndrome - it is well known the sodium channels are temperature sensitive. Fever can block sodium channels, which can result in worsening of the Brugada pattern on ECG and increase the risk of sudden death in patients with a type I Brugada pattern. For these reasons, fever in patients with Brugada syndrome and COVID-19, should be treated promptly with paracetamol.(12)

\section{TRIAGE AND MANAGEMENT OF ARRHYTHMIAS AND OTHER ELECTROPHYSIOLOGY PROCEDURES DURING THE COVID-I 9 PANDEMIC}

The principles underlying the management of patients with cardiac arrhythmias during the COVID-19 pandemic are largely governed by the need to limit the peri-procedural COVID-19 exposure for both the EP team and the patients. ${ }^{(8)}$ This necessitates the categorisation of EP procedures into elective, urgent or emergent. All elective ablation and cardiac device implantation procedures should be postponed, and antiarrhythmic medications should be reviewed and intensified if necessary, to allow control of symptomatic arrhythmia recurrences during the COVID- 19 pandemic period. CIED follow-up should be done via remote monitoring in all patients, where possible (2) $^{(2)}$

Procedures can be classified as summarised in Table $\mid:(2,8)$

\section{PROTECTING STAFF AND THE APPROPRIATE USE OF PERSONAL PROTECTIVE EQUIPMENT (PPE)}

When treating patients during the COVID-19 pandemic, it is important to be aware of the risk of exposure to the EP team, during both consultations and invasive procedures. As already mentioned, face-to-face consultations should be limited and many consultations can be done by, for example, a review of the patient's chart and ECGs. ${ }^{(8)}$ When community spread is high, it is prudent to consider all patients potentially infected with COVID- 19 and to take appropriate precautions to protect the healthcare workers. Guidelines for the use of PPE are likely to differ slightly depending on the institution and the availability of PPE, but some basic principles are common to all. When considering the use of PPE, one needs to consider both the patient being treated and the procedure being done, with the highest risk being an aerosol-generating procedure in a known COVID- 19 infected patient, and the lowest risk being a simple clinical examination in a known COVID negative patient.

Most institutions have guidelines for the use of PPE stratified by the combined risk. There are general measures which should be taken in all situations, and specific guidelines for PPE in addition to these general measures.

\section{General measures to prevent infection ${ }^{(13)}$}

\section{Hand hygiene}

Transmission can be greatly reduced by an increased frequency of hand hygiene using the "World Health Organisation (WHO) 5 Moments for Hand Hygiene":

Wash hands with soap and water for 20 seconds or use alcohol-based hand sanitiser after each patient contact and after contact with frequently touched surfaces (e.g. door handles, keyboards, phones, work surfaces).

Respiratory etiquette: cough and sneeze into a flexed elbow or into a tissue (immediately discard the soiled tissue in a bin and wash hands). 
TABLE I: Risk stratification of electrophysiology procedures.

\begin{tabular}{|c|c|c|c|}
\hline & Emergency & Urgent & Elective \\
\hline Catheter ablation & $\begin{array}{l}\text { VT ablation for electrical storm } \\
\text { AF/AFL with tachycardia induced } \\
\text { cardiomyopathy, refractory to medical } \\
\text { therapy including cardioversion } \\
\text { WPW syndrome with fast } \\
\text { pre-excited AF }\end{array}$ & $\begin{array}{l}\text { VT ablation for medically } \\
\text { refractory recurrentVT } \\
\text { AF/AFL ablation for medically refractory } \\
\text { AF/AFL (including cardioversion), with } \\
\text { recurrent emergency room visits } \\
\text { SVT ablation for medically refractory } \\
\text { SVT with recurrent emergency } \\
\text { room visits }\end{array}$ & $\begin{array}{c}\text { PVC ablation } \\
\text { AF/AFL ablation } \\
\text { SVT ablation/diagnostic EP testing }\end{array}$ \\
\hline CIED procedures & $\begin{array}{l}\text { Generator change in a patient where } \\
\text { EOL present or imminent } \\
\text { Secondary prevention ICD } \\
\text { PM for high degree AVB or SND } \\
\text { with long pauses } \\
\text { CRT implant in a patient with severe, } \\
\text { medically refractory heart failure } \\
\text { Lead revision with lead malfunction in a } \\
\text { pacemaker-dependent patient or in a } \\
\text { patient with an ICD receiving } \\
\text { inappropriate shocks } \\
\text { Lead extraction for infection }\end{array}$ & $\begin{array}{l}\text { Generator change in a patient } \\
\text { where ERI present } \\
\text { Primary prevention ICD in patient } \\
\text { with very high risk of arrhythmias }\end{array}$ & $\begin{array}{c}\text { Generator change in a patient where } \\
\text { ERI >6 weeks } \\
\text { Primary prevention ICD } \\
\text { PM for SND, Mobitz I AVB, stable } \\
\text { non-high degree AVB, or tachy-brady } \\
\text { syndrome in mildly symptomatic patient } \\
\text { CRT implant or device upgrade } \\
\text { in stable patient } \\
\text { Lead revision for } \\
\text { asymptomatic malfunction } \\
\text { Lead extraction in a patient } \\
\text { without infection }\end{array}$ \\
\hline Other & $\begin{array}{l}\text { Cardioversion of medically refractory } \\
\text { atrial arrhythmias with rapid } \\
\text { ventricular rates } \\
\text { TOE for urgent cardioversion }\end{array}$ & & $\begin{array}{l}\text { Cardioversion of stable arrhythmias } \\
\text { with well tolerated symptoms } \\
\text { TOE for routine assessment of valves or } \\
\text { LAA closure devices and cardioversion } \\
\text { that can be done after appropriate } \\
\text { period of anticoagulation } \\
\text { ILR implantation } \\
\text { Tilt table testing } \\
\text { LAA closure } \\
\text { Ambulatory rhythm monitoring }\end{array}$ \\
\hline
\end{tabular}

$V T=$ ventricular tachycardia, $P V C=$ premature ventricular complex, $A F=$ atrial fibrillation, $A F L=$ atrial flutter, WPW $=$ Wolff-Parkinson-White, SVT $=$ supraventricular tachycardia $E P=$ electrophysiology, $C I E D=$ cardiovascular implantable electronic device, $E O L=$ end of life, $E R I=$ elective replacement indicator, ICD $=$ implantable cardioverter-defibrillator, $P M=$ pacemaker, $A V B=$ atrio-ventricular block, SND = sinus node disease, CRT = cardiac resynchronisation therapy, TOE = tranoesophageal echocardiogram, LAA = left atrial appendage, ILR = implantable loop recorder.

- Avoid touching your mouth, eyes and nose with gloved or bare hands. Wash hands before doing so.

Avoid physical contact such as handshakes and hugs.

- Social distancing: maintain a distance of at least 1.5 - 2 metres away from other people.

Environmental cleaning: frequently touched surfaces and equipment in clinical areas should be cleaned and disinfected twice daily (minimum), and after every use.

- Work clothes: Remove your work clothes at the workplace and place in a plastic bag to take home, and clean with a warm wash. Or, remove your work clothes immediately upon arriving home, and clean with a warm wash. Always wash your hands after removing your work clothes.
Types of PPE and the clinical situations in which they should be applied:(2,9,13)

This is summarised in Table II.

All healthcare workers should familiarise themselves with the correct donning and doffing procedures (aided by a donning and doffing buddy system and visible posters where possible).

Recommendations for invasive procedures: ${ }^{(2,8,14)}$

Emergency and urgent invasive electrophysiology procedures are often performed in patients at high risk of haemodynamic instability, cardioversion and potentially cardio-pulmonary resuscitation.

- As such, the recommendation is that full PPE be donned for these procedures. 
TABLE II: Recommendations for the use of PPE.

\begin{tabular}{|c|c|c|c|c|c|}
\hline $\begin{array}{l}\text { Type of } \\
\text { PPE }\end{array}$ & $\begin{array}{l}\text { Clinical staff } \\
\text { (nurses, doctors, EMS) } \\
\text { providing direct care } \\
\text { to COVID-19 patients } \\
\text { or patients with } \\
\text { respiratory symptoms }\end{array}$ & $\begin{array}{c}\text { Non-clinical } \\
\text { staff } \\
\text { (admin staff, catering } \\
\text { staff) coming into } \\
\text { distant contact with } \\
\text { COVID- } 19 \text { patients } \\
\text { and contaminated } \\
\text { surfaces }\end{array}$ & $\begin{array}{c}\begin{array}{c}\text { Non-clinical } \\
\text { staff }\end{array} \\
\text { (cleaners) coming into } \\
\text { distant contact with } \\
\text { COVID-19 patients } \\
\text { and contaminated } \\
\text { surfaces }\end{array}$ & $\begin{array}{l}\text { Patients } \\
\text { with respiratory } \\
\text { symptoms }\end{array}$ & $\begin{array}{c}\text { Patients } \\
\text { without respiratory } \\
\text { symptoms }\end{array}$ \\
\hline Gloves & $\begin{array}{l}\text { Non-sterile gloves } \\
\text { Change between } \\
\text { patients }\end{array}$ & $\begin{array}{l}\text { Non-sterile gloves } \\
\text { Change when leaving } \\
\text { COVID-19 area }\end{array}$ & $\begin{array}{l}\text { Reusable long rubber } \\
\text { utility cleaning gloves } \\
\text { (ideally up to elbow) } \\
\text { Change after completed } \\
\text { cleaning contaminated } \\
\text { area }\end{array}$ & None & None \\
\hline Masks & $\begin{array}{c}\text { Surgical mask } \\
\text { for general care of } \\
\text { COVID-19 patients } \\
\text { N95 respirator for } \\
\text { aerosol-generating } \\
\text { procedures on } \\
\text { COVID-19 } \\
\text { suspects/cases }\end{array}$ & $\begin{array}{l}\text { Surgical mask } \\
\text { when within }<1 \mathrm{~m} \text { of a } \\
\text { patient with respiratory } \\
\text { symptoms (one per shift, } \\
\text { if integrity maintained) }\end{array}$ & $\begin{array}{c}\text { Surgical mask } \\
\text { when within }<1 \mathrm{~m} \text { of a } \\
\text { patient with respiratory } \\
\text { symptoms }\end{array}$ & $\begin{array}{c}\text { Surgical mask } \\
\text { worn when in contact } \\
\text { with others }\end{array}$ & None \\
\hline Aprons & $\begin{array}{l}\text { Change when visibly } \\
\text { contaminated } \\
\text { Discard after aerolisation } \\
\text { procedure }\end{array}$ & $\begin{array}{l}\text { Change when leaving } \\
\text { COVID-19 area }\end{array}$ & $\begin{array}{l}\text { Change after each work } \\
\text { session (in absence of } \\
\text { clinical contact) }\end{array}$ & None & None \\
\hline Eye cover & $\begin{array}{c}\text { Face shield/goggles } \\
\text { Wash clean, disinfect } \\
\text { and reuse }\end{array}$ & None & $\begin{array}{c}\text { Face shield/goggles } \\
\text { Wash clean, disinfect } \\
\text { and reuse }\end{array}$ & None & None \\
\hline
\end{tabular}

$E M S=$ emergency medical services.

Full PPE in the cardiac catheterisation laboratory includes a sterile gown, double gloves and shoe covers.

\section{POTENTIAL THERAPIES FOR COVID-I 9 AND THE RISK OF QT PROLONGATION AND TORSADE DE POINTES}

When the new disease caused by COVID-19 started to appear, initially in late 2019 in China, and subsequently in the rest of the world from early 2020 onwards, much emphasis was placed on finding therapies to prevent, modulate and potentially treat this new problem. A lot of the early work came from China and suggested that hydroxychloroquine/chloroquine together with azithromycin might be very effective in this regard, but immediately cardiologists, and especially those dealing with arrhythmias, became extremely concerned about the risk of malignant pro-arrhythmias due to the QT-prolonging effects of these drugs individually, and especially in combination, with the risk of TdP. Almost immediately, rhythm societies from around the world published guidelines with regard to the use of potentially QT-prolonging drugs and guidelines with regard to assessment in this regard, with the American societies including the Heart Rhythm Society, together with the American College of Cardiology and the American Heart Association guidelines probably being the most prominent. ${ }^{(15)}$

However, a number of other drugs used in the treatment of patients with COVID-19, and especially those who are seriously ill, also have possible QT-prolonging effects. Unfortunately, underlying cardiovascular disease and hypertension also significantly increase the risk of serious COVID-19 related disease, in a population already at risk of significant arrhythmias. In addition, one has to remember that the disease itself is associated with significant direct cardiovascular effects, particularly myocarditis, and that some of these patients will become seriously ill, which in itself predisposes to both atrial and ventricular tachyarrhythmias and to bradyarrhythmias. Furthermore, the acute systemic inflammatory response seen in these patients also significantly increases the risk of arrhythmias, and the combination of QT-prolonging drugs and the serious underlying comorbidities combined significantly increases the risk of malignant arrhythmias. 
It would seem as though the initial enthusiasm for the combination of hydroxychloroquine/chloroquine and azithromycin brought about by early Chinese publications and reports and the French studies and other reviews suggesting decreased viral load and potential clinical benefit, have not been borne out. ${ }^{(16-18)}$ More recent studies both from North and South America, and publications from registries in high-profile journals have in fact suggested that there were significant levels of QT prolongation and an increased risk of arrhythmias - with efficacy not really borne out. ${ }^{(5,19,20)}$ In many countries these drugs have therefore been withdrawn from guidelines, but have remained in the guidelines of a number of countries with some highprofile figures worldwide, with these recently also espousing the use of these drugs.

A number of other drugs have however been suggested as possible therapies for COVID-19, including the antiviral agent remdesivir, the HIV drugs lopinavir and ritonavir, the influenza drug favipravir, together with ribavirin and interferon beta Ib, and other agents to modify the systemic inflammatory response. ${ }^{(21)}$ A number of these are presently in various states of investigation with regard to their usefulness and efficacy in the management of COVID-19. A significant number of these have also been associated with, or are potentially implicated in QT segment prolongation, with arrhythmias noted as sideeffects of some of these drugs.

One also has to remember that some of the agents used in patients on ventilators for sedation and in ill patients for nausea, together with a number of antibiotics, have also been implicated in QT segment prolongation and therefore combination therapies have to be looked at extremely carefully in patients on polypharmacy to try and prevent the use of multiple QT-prolonging drugs.

Keeping track of all of the potential QT-prolonging drugs can be very problematic and therefore we would recommend the use of a website and smart phone application specifically developed in this regard, https://www.crediblemeds.org/ and the crediblemeds app. Both of these are kept up-to-date on a regular basis, particularly currently.

Calculation of the QT interval itself has been shown to be poorly performed in general, even by experienced people, and is therefore difficult, and automated ECG algorithms are often incorrect. ${ }^{(22)}$ It is important to note that the QT should be calculated from the beginning of the QRS complex and using the tangent method to calculate the end of the $T$ wave, and then corrected using the preceding RR interval, using I of the standard calculations, most commonly Bazett's formula $\mathrm{QTc}=\mathrm{QT} /$ square root of the preceding RR interval - see
Figure I. Some averaging over a number of beats may be needed, especially when the rhythm is irregular, and calculating the QT when the QRS complex is broad is also challenging.

One challenge in correcting the QT, particularly in ill patients with sinus tachycardia, can be choosing a correction formula. The most common correction formula, Bazett's formula, is inaccurate at heart rates less than 60 or greater than II 0bpm and has a tendency to overestimate the QTc, possibly leading to unnecessary discontinuation of therapy. Alternative formulas have shown better accuracy and less heart rate dependency, but can be more tricky to calculate.

\section{The North American guidelines suggest: ${ }^{(15)}$}

\section{Electrocardiographic/QT interval monitoring;}

- Withhold hydroxychloroquine and azithromycin in patients with baseline QT prolongation (e.g. QTc $\geq 500 \mathrm{~ms}$ ) or with known congenital long QT syndrome;

- Monitor cardiac rhythm and QT interval; withdrawal of hydroxychloroquine and azithromycin if QTc exceeds a present threshold of $500 \mathrm{~ms}$;
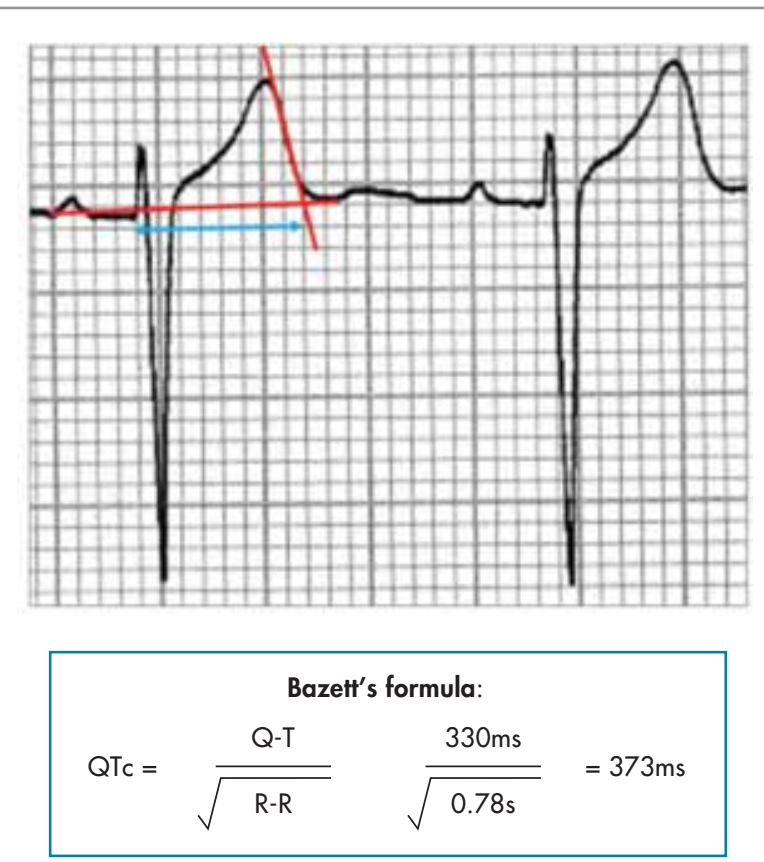

FIGURE I: The QT interval should be calculated from the beginning of the QRS complex and then use the tangent method to calculate the end of the T wave, and then corrected using the RR interval and the Bazett's formula. In this example, a tangent line is drawn with the downslope of the T wave, and the end of the QT interval is taken where it intersects the isoelectric line. The preceding R-R interval of the measured QT interval should be used in the calculation. 
In patients critically ill with COVID-19 infection, frequent caregiver contact may need to be minimised, so optimal electrocardiographic interval and rhythm monitoring may not be possible; in these cases, some have suggested that the use of telemetry may be used as a compromise to prevent frequent caregiver contact

- Correction of hypokalaemia $>4 \mathrm{mmol} / \mathrm{L}$ and hypomagnesemia $>2 \mathrm{mg} / \mathrm{dL}$; and

Avoid other QTc-prolonging agents, whenever feasible.

Further experience going forward will undoubtedly yield further information with regard to the risk of arrhythmias, with therapies being evaluated for the treatment of COVID-19. It is also appropriate to review how to accurately measure the QT interval and to remember the potential QT-prolonging effects of many of the drugs that we use. Furthermore, it may prompt us to look more carefully at some therapies that are known to prolong the QT interval where ECG monitoring is not undertaken, prior to its initiation and during the course of treatment.

\section{CONSIDERATIONS FOR THE USE OF ANTIARRHYTHMIC DRUGS AND ANTICOAGULATION IN PATIENTS WITH COVID-I 9}

When using antiarrhythmic drugs in these patients, standard guidelines should apply, always remembering that the antiarrhythmic drugs themselves have proarrhythmic effects and in many cases can themselves significantly prolong the QT interval.

In terms of oral anticoagulation, it is inevitable that many patients who develop severe COVID- 19 related disease will already have been on oral anticoagulation, either with vitamin $\mathrm{K}$ antagonists or with direct oral anticoagulants. In addition, there is increasing evidence that COVID-19 promotes thrombosis and that this mechanism may be a significant contributor to both morbidity and mortality. ${ }^{(23,24)}$ In this regard, a number of trials have been initiated looking at the use of anticoagulation, predominantly in the form of low molecular weight heparin, in the management of these patients. A recently published research letter from New York has elicited some controversy, but suggested that in patients requiring mechanical ventilation, in-hospital mortality and overall survival was significantly improved using a combination of heparin, low molecular weight heparin and direct oral anticoagulants. ${ }^{(25)}$ This was a retrospective study and was not randomised and further randomised trials are ongoing. Of note is that an elevated risk of bleeding events associated with oral anticoagulation has been highlighted in a number of studies. One recent publication suggested that patients on vitamin $\mathrm{K}$ antagonists who are hospitalised with
COVID-I 9 show high instability of INR measurements probably related to alterations in vitamin $\mathrm{K}$ metabolism, together with polypharmacy and renal and liver dysfunction. ${ }^{(26)}$ In addition, there may be significant pharmacological interactions with the direct oral anticoagulants.

At present it would seem as though anticoagulation should be carefully reassessed in those who present with COVID- 19 and who are on oral anticoagulation, particularly regarding how this is administered and monitored, but that some form of anticoagulation may well be beneficial in patients with significant COVID-1 9 disease. Again, further information will undoubtedly be forthcoming in this regard in the short to medium term.

\section{CONCLUSION}

Arrhythmias can complicate the clinical course of patients with COVID- 19 infection in a significant number of patients. Management of arrhythmias should generally follow standard guidelines of arrhythmia management. Catheter ablation in the midst of the COVID-19 pandemic should be limited to arrhythmias that are immediately life-threatening or which may affect prognosis in the short term. Numerous COVID-19 therapies are under investigation at present. Currently no therapy has shown to be effective in the treatment of COVID-19 and some may prolong the QT interval which may predispose patients to lifethreatening arrhythmias. Careful monitoring of QTc intervals is essential in patients at risk and potential therapies that prolong the QT interval should be restricted to clinical trials.

\section{Conflict of interest: none declared.}


I. Johns Hopkins Coronavirus Resource Center. Accessed on I June 2020.

2. European Society of Cardiology. ESC Guidance for the Diagnosis and Management of CV Disease during the COVID-19 Pandemic. Retrieved: https://www.escardio.org/Education/COVID-19-and-Cardiology/ESCCOVID-19-Guidance. Published in 2020.

3. Liu W, Tao ZW, Wang L, et al. Analysis of factors associated with disease outcomes in hospitalised patients with 2019 novel coronavirus disease. Chinese Med J-Peking 2020; 133(9): 1032-1038.

4. Wang DW, Hu B, Hu C, et al. Clinical characteristics of 138 hospitalised patients with 2019 novel coronavirus-infected pneumonia in Wuhan, China. J Am Med Assoc 2020;323(1 1): 1061-1069.

5. The reference has been retracted from the Lancet.

6. Driggin E, Madhavan MV, Bikdeli B, et al. Cardiovascular considerations for patients, health care workers, and health systems during the COVID-19 pandemic. J Am Coll Cardiol 2020;75( ( 8):2352-237।.

7. Lazzerini PE, Boutjdir M, Capecchi PL. COVID-19, arrhythmic risk and inflammation: Mind the gap! Circulation 2020.

8. Lakkireddy DR, Chung MK, Gopinathannair R, et al. Guidance for cardiac electrophysiology during the coronavirus (COVID-19) pandemic from the heart rhythm society COVID-19 task force; Electrophysiology Section of the American College of Cardiology; and the Electrocardiography and Arrhythmias Committee of the Council on Clinical Cardiology, American Heart Association. Heart Rhythm 2020.

9. Jain S, Workman V, Ganeshan R, et al. Enhanced ECG monitoring of COVID-19 patients. Heart Rhythm 2020.

10. Prutkin JM. Coronavirus disease 2019 (COVID-19): Arrhythmias and conduction system disease. UpToDate 2020: I - 14

1 1. Wu Cl, Postema PG, Arbelo E, et al. SARS-CoV-2, COVID-19, and inherited arrhythmia syndromes. Heart Rhythm 2020.

12. Offerhaus JA, Bezzina CR, Wilde AAM. Epidemiology of inherited arrhythmias. Nat Rev Cardiol 2020;17(4):205-215.

13. Mehtar S. COVID-19 infection prevention and control guidelines for South Africa - Draft VI. Retrieved: https://www.sapc.za.org/Media/Default/ Documents/Final draft COVID- 19 IPC Guidelines 31 MARCH.pdf.

14. John TJ, Hassan K, Weich H. Donning and doffing of personal protective equipment (PPE) for angiography during the COVID-19 crisis. Eur Heart J 2020;4I (19): 1786-1787

15. Roden DM, Harrington RA, Poppas A, et al. Considerations for drug interactions on QTc interval in exploratory COVID-19 treatment. J Am Coll Cardiol 2020;75(20):2623-2624.

16. Yao $X$, Ye F, Zhang $M$, et al. In vitro antiviral activity and projection of optimised dosing design of hydroxychloroquine for the treatment of Severe Acute Respiratory Syndrome Coronavirus 2 (SARS-CoV-2). Clin Infect Dis 2020.

17. Colson P, Rolain JM, Lagier JC, et al. Chloroquine and hydroxychloroquine as available weapons to fight COVID-19. Int J Antimicrob Agents 2020;55(4): 105932

18. Cortegiani A, Ingoglia G, Ippolito M, et al. A systematic review on the efficacy and safety of chloroquine for the treatment of COVID-19. J Crit Care 2020;57:279-283

19. Geleris J, Sun Y, Platt J, et al. Observational study of hydroxychloroquine in hospitalised patients with Covid-19. N Engl J Med 2020.

20. Borba MGS, Val FFA, Sampaio VS, et al. Effect of high vs low doses of chloroquine diphosphate as adjunctive therapy for patients hospitalised with Severe Acute Respiratory Syndrome Coronavirus 2 (SARS-CoV-2) infection: A randomised clinical trial. JAMA Netw Open 2020;3(4):e208857.

21. Goldman JD, Lye DCB, Hui DS, et al. Remdesivir for 5 or 10 days in patients with severe Covid-19. N Engl J Med 2020.
22. Viskin S, Rosovski U, Sands AJ, et al. Inaccurate electrocardiographic interpretation of long QT: The majority of physicians cannot recognise a long QT when they see one. Heart Rhythm 2005;2(6):569-274.

23. Tang $\mathrm{N}$, Bai $\mathrm{H}$, Chen $\mathrm{X}$, et al. Anticoagulant treatment is associated with decreased mortality in severe coronavirus disease 2019 patients with coagulopathy. J Thromb Haemost 2020; 18(5): 1094- 1099.

24. Thachil J, Tang N, Gando S, et al. ISTH interim guidance on recognition and management of coagulopathy in COVID-19. J Thromb Haemost 2020; 18(5): 1023-1026.

25. Paranjpe I, Fuster V, Lala A, et al. Association of treatment dose anticoagulation with in-hospital survival among hospitalised patients with COVID-19. J Am Coll Cardiol 2020.

26. Testa S, Paoletti O, Giorgi-Pierfranceschi M, et al. Switch from oral anticoagulants to parenteral heparin in SARS-CoV-2 hospitalised patients. Intern Emerg Med 2020. 